\title{
A Stochastic Model of Gene Regulation Using the Chemical Master Equation
}

\author{
Hilary S. Booth, ${ }^{1}$ Conrad J. Burden, ${ }^{2,3}$ Markus Hegland, ${ }^{4,5}$ and Lucia Santoso ${ }^{2,5}$ \\ 1 This paper is dedicated to Dr. Hilary Booth who died unexpectedly on May 26, 2005. \\ 2 Centre for Bioinformation Science, Mathematical Sciences Institute, Australian National \\ University, Canberra, A.C.T. 0200, Australia; conrad.burden@anu.edu.au \\ 3 John Curtin School of Medical Research, Australian National University, Canberra, A.C.T. \\ 0200, Australia \\ 4 Centre for Mathematics and Its Applications, Mathematical Sciences Institute, Australian \\ National University, Canberra, A.C.T. 0200, Australia \\ 5 ARC Centre in Bioinformatics, The University of Queensland, St. Lucia, QLD. 4072, \\ Australia
}

Summary. The chemical master equation in combination with chemical rate equations is used as a tool to study Markovian models of genetic regulatory networks in prokaryotes. States of the master equation represent the binding and unbinding of protein complexes to DNA, resulting in a gene being expressed or not expressed in a cell, while protein and substrate concentrations are represented by continuum variables which evolve via differential equations.

The model is applied to a moderately complex biological system, the switching mechanism of the Bacteriophage $\lambda$ driven by competition between production of $\mathrm{CI}$ and Cro proteins. Numerical simulations of the model successfully move between lysogenic and lytic states as the host bacterium is stressed by the application of ultraviolet light.

Key words: Gene regulation, chemical master equation, bacteriophage lambda.

AU: is "Bacteriophage" always capitalized, and should it be italicized?

\subsection{Modelling Gene Regulatory Networks}

One of the major challenges in bioinformatics is to determine how genes are regulated and how their products interact within cellular networks. In a complex cell, gene products and external factors regulate the genes that are expressed in that cell. Some gene products promote or repress other genes, usually depending on the concentration of the gene product. In some cases two genes compete for expression, resulting in a population of cells distributed between the competing states. The expression levels of other genes are affected by environmental parameters such as temperature or UV light, sometimes via the degradation rate of the protein that regulates the gene expression, e.g., the $c I$ and cro genes of the Bacteriophage $\lambda$ [1].

The challenge is to model the complex genetic componentry that enables a cell to switch genes "on" and "off" as required [2,3]. Small models have been developed to 
describe gene promotion and repression [4], and these components can be combined into more complex models [5-7]. In some cases, artificial genetic machines based upon well-understood genetic components have been constructed and their behaviour has been analysed in a more controlled environment $[8,9]$. Some biologically stable states and bistable systems can be modelled using stochastic differential equations that describe the protein levels as functions of the gene states $[5,10]$.

This chapter discusses the mathematical modelling of gene regulation in the Bacteriophage $\lambda$. Reflecting the stochastic nature of gene regulation, the chemical master equation is used as a tool to study Markovian models of networks of gene states between which probabilistic transitions occur. These states represent the binding or unbinding of protein complexes to DNA, resulting in a gene being expressed or not expressed in the cell. In Section 7.2 the stochastic master equation for an arbitrary network is given. We also describe a generic hybrid model which combines deterministic chemical rate equations with a stochastic model of gene switching.

In Section 7.3 our hybrid model is applied to a simplified version of the switching mechanism of the phage $\lambda$ as it moves stochastically between its alternate lifestyles (lysis or lysogeny). A stochastic simulation in Section 7.4 shows the phage $\lambda$ evolving through a set of states. At each time step the virtual phage $\lambda$ chooses a new state and, depending upon that state, $\mathrm{CI}$ and Cro proteins are produced. These proteins compete with each other to reach one of the two alternative lifestyles of the phage $\lambda$. The application of UV light to the host bacterium is also simulated and its effect is shown on the outcome of the competition between CI and Cro.

\subsection{Stochastic Master Equation Model of Gene Regulation}

The stochastic formulation of gene regulation is based on an assumption that the underlying chemical processes are Markovian. An efficient tool for dealing with Markovian processes is the stochastic chemical master equation [11]. In this formalism, a regulatory network is typically represented in a state space, elements of which describe the states or abundances of a finite number of chemical species which may be made up of any combination of genes, RNA, proteins or substrates. The gene states, for example, may be defined as gene on/off, i.e., the gene is/is not being expressed in the cell. Alternatively, we may wish to specify that a protein or enzyme is attached/not attached to a promoter or operator site, and also specify the absolute protein levels measured in numbers of molecules or in concentrations.

Consider a system that can be in any one of a finite number of states $s=1, \ldots, N$, and capable of making transitions $i=1, \ldots, N_{T}$ between states. Since the system is Markovian, the probability of making a transition at any given time depends only on the state of the system at that time, and not on its history. We represent the system by a directed graph with $N$ vertices and $N_{T}$ edges. Associated with each transition is a propensity $\alpha_{i}>0$. If $i$ is the transition from state $s$ to state $r$, the probability of making the transition $i$ in the time interval $[t, t+d t)$, conditional on being in state $s$ at time $t$, is $\alpha_{i} d t$. Given an initial probability distribution among the states of $\mathbf{p}(0)=$ $\left(p_{1}(0), \ldots, p_{N}(0)\right)^{\mathrm{T}}$, with $p_{s}>0$ and $\sum_{s} p_{s}=1$, the system evolves in time to a 
distribution $\mathbf{p}(t)=\left(p_{1}(t), \ldots, p_{N}(t)\right)^{\mathrm{T}}$ via the stochastic master equation

$$
\frac{d \mathbf{p}}{d t}=A \mathbf{p}(t),
$$

where the matrix $A$ is given in terms of propensities. If $\alpha_{s r}$ is the propensity associated with the edge running from vertex $s$ to vertex $r$, then

$$
A=\sum_{s, r} \alpha_{s r} \Pi_{s r},
$$

where the matrices $\Pi_{s r}$ are defined by

$$
\Pi_{s r}=\left(\begin{array}{cccccc}
0 & & & & & \\
& \ddots & & & & \\
& & -1 & & & \\
& & \vdots & \ddots & & \\
& +1 & & \ddots & \\
& & & & & 0
\end{array}\right),
$$

with -1 in the $s$ th row and column to account for the flow of probability away from the $s$ th state, +1 in the $r$ th row and the $s$ th column to account for the flow of probability into the $r$ th state, and all other entries zero. Because each column of $\Pi_{s r}$ sums to zero, so does each column of $A$. One easily checks that this is equivalent to saying that total probability is conserved: $d\left(\sum_{s} p_{s}(t)\right) / d t=0$. For a specified initial condition, and with constant propensities, the solution to Eq. (7.1) is given formally by

$$
\mathbf{p}(t)=e^{t A} \mathbf{p}(0) .
$$

For any realistic system, the dimensionality of the state space soon becomes prohibitively large if the concentration of all chemical species (proteins and enzymes for instance) and the binding of protein complexes to operator sites are to be described. To see this, consider that every possible combination of concentrations and operator binding states is represented by a different basis vector of the space in which the probability vector $\mathbf{p}(t)$ evolves. To reduce the problem to manageable proportions, we propose here a hybrid stochastic/deterministic model in which the evolution of continuum concentrations $\mathbf{x}(t)=\left(x_{1}(t), x_{2}(t), \ldots, x_{n}(t)\right)$ of chemical species is modelled using deterministic chemical rate equations, and the switching between operator binding states $s=1, \ldots, N$ is modelled using the stochastic master equation. In general, the chemical rate equations will be differential equations of the form

$$
\frac{d x_{i}}{d t}=f_{i}(\mathbf{x}, s),
$$

and the propensities $\alpha_{r s}(\mathbf{x})$ will depend on chemical concentrations, since the probability that a given protein complex will bind to a particular operator site will depend on the concentration of that protein complex. 
In principle, the system evolves over infinitesimal time steps $d t$ along a path $(\mathbf{x}(t), s(t))$, where

$$
x_{i}(t+d t)=x_{i}(t)+f_{i}(\mathbf{x}(t), s(t)) d t
$$

and $s(t+d t)$ is drawn from the probability distribution resulting from the action of the matrix $I+A(s) d t$ on the distribution concentrated at the state $s(t)$.

In practice, our numerical simulations use small finite time steps $\Delta t$ over which the protein concentrations and protein binding state are evolved. The choice of $\Delta t$ is crucial to the success of the model. Gene regulatory systems in prokaryotes typically have two intrinsic time scales: a slow scale set by chemical rate constants over which protein and substrate concentrations change, and a fast time scale over which gene switching driven by the binding of proteins to operators occurs. By choosing $\Delta t$ intermediate between these scales, the essential dynamics can be efficiently captured. In the simulations that follow, protein concentrations are evolved using a Runge-Kutta algorithm with the state $s$ set to its value at the beginning of the time interval $\Delta t$. At the end of each time step, a new operator binding state $s(t+\Delta t)$ is chosen from a distribution obtained by evolving Eq. (7.4) over the finite $\Delta t$ using propensities set to their values at the beginning of the time step and a very accurate approximation to the matrix exponential times the distribution $p_{r}(t)=\delta_{r s(t)}$ concentrated at the state $s(t)$. We note that this model is not suitable for modelling regulation in eukaryotes, in which transcription can be regulated over much slower time scales.

\subsection{The Switching Mechanism of the Bacteriophage $\lambda$}

An example of a genetically regulated biological switching system is the Bacteriophage $\lambda$. The phage $\lambda$ invades a bacteria cell (E. coli), after which it can enter into one of two alternative lifestyles called lysogeny and lysis [1]. The lysogeny stage is a dormant stage in which the phage inserts its DNA into the host's DNA and passively reproduces with the host. When the host becomes stressed, the phage is more likely to go into lysis, in which case it reproduces more phages, kills the host and spreads to other bacteria cells. The decision between lysis and lysogeny can be thought of as a switching mechanism. The stochastic switch is based upon a competition between two genes in the phage's DNA: cro and $c I .{ }^{6}$ If the production of Cro proteins dominates, the system enters the lytic stage. If the production of CI proteins dominates, the system remains in the lysogenic stage.

Fig. 7.1 shows the section of phage $\lambda$ DNA containing the cro and $c I$ genes. The intervening region contains three operators $O_{R 1}, O_{R 2}$ and $O_{R 3}$ which control the promoters $P_{R}$ and $P_{R M}$. The gene products readily dimerise to form complexes $\mathrm{Cro}_{2}$ and $\mathrm{CI}_{2}$ which can bind to any of the three intervening operator sites. $\mathrm{CI}_{2}$ dimers bind preferentially to $O_{R 1}$ and $O_{R 2}$, but can also bind to $O_{R 3}$, with a lower probability. $\mathrm{Cro}_{2}$ dimers, on the other hand, bind preferentially to $O_{R 3}$, but can also bind to $O_{R 1}$ and

\footnotetext{
${ }^{6}$ Capitalised names such as CI and Cro are used to represent proteins, while italicized names,
} such as $\mathrm{CI}$ and $\mathrm{cro}$ are used to represent the genes which encode for them. 


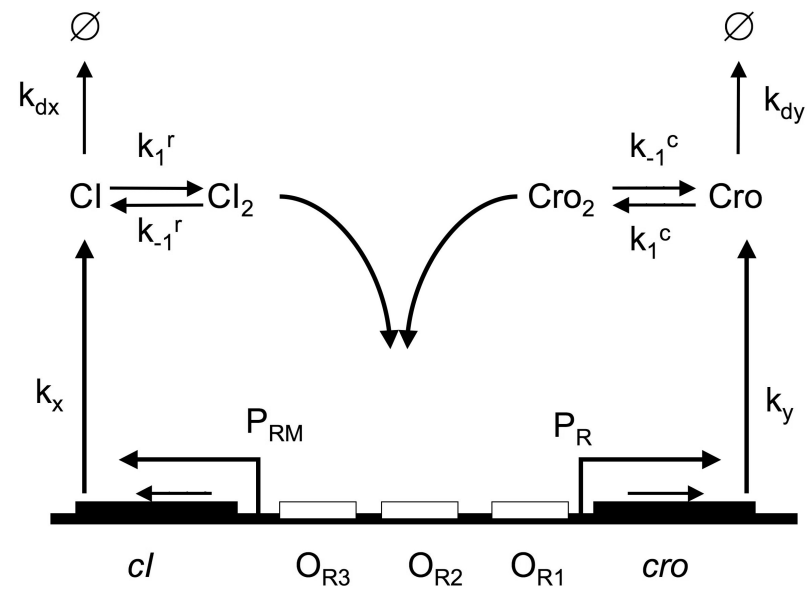

Fig. 7.1. The switching mechanism of the Bacteriophage $\lambda$ in which $c I$ and cro genes compete stochastically for expression.

$O_{R 2}$. If either $O_{R 1}$ or $O_{R 2}$ is occupied, RNA polymerase is prevented from binding to the cro promoter $P_{R}$, and production of Cro protein ceases. Likewise, if $O_{R 3}$ is occupied, the promoter $P_{R M}$ is blocked and production of CI protein ceases. Thus, at low concentrations both genes enhance their own production by inhibiting the production of the competing protein, and at high concentrations repress their own production. Also represented in Fig. 7.1 are decays of $\mathrm{CI}$ and Cro monomers.

The full story of the lysis/lysogeny decision mechanism is considerably more complex than the simplified version given here. See [1] for a full biological description, and [12] for a comprehensive stochastic model that includes the other proteins (CII, $\mathrm{CIII}$ and $\mathrm{N}$ ) involved in the process, as well as the production of mRNA, its translation into proteins, the degradation rates of all chemical species and cell division.

The hybrid model we describe below differs from the previous model of Reinitz and Vaisnys [13] in our use of the stochastic master equation to introduce noise into the system via the switching between operator binding states. In contrast, Reinitz and Vaisnys assume operator binding states to be in chemical equilibrium, from which they estimate a time averaged protein synthesis rate. This leads to a deterministic set of coupled differential equations. There exist models which include noise into the differential equations by arbitrarily introducing additive or multiplicative stochastic terms to protein synthesis or decay rates $[10,14]$. However, we are unaware of any hybrid treatment in which substrate concentrations are determined by chemical rate equations, but the operator binding is determined using the stochastic master equation. The highly sophisticated model of Arkin et al. [5] is a simulation of the stochastic master equation using the Gillespie algorithm [15] to model all aspects of protein and RNA synthesis, including translation and transcription of genes, and decay in the phage $\lambda$ system. Such models are a valuable and necessary contribution but are unfortunately computationally expensive and not easily adaptable to more complex regulatory networks. The model we propose here is designed to be computationally efficient and easily adapt- 
able while still retaining the important characteristics of a moderately complex genetic system.

\subsubsection{The Model}

Following the conventions of Ref. [13], we indicate the operator binding states of the system by a discrete variable $s=\{1, \ldots, 40\}$. This variable represents the 40 ways in which either $\mathrm{CI}_{2}$ or $\mathrm{Cro}_{2}$ dimers can bind to $O_{R 1}, O_{R 2}$ and $O_{R 3}$, and RNA polymerase can bind to the promoters $P_{R}$ and $P_{R M}$ subject to the constraints that $P_{R}$ cannot be occupied if either $O_{R 1}$ or $O_{R 2}$ is occupied and $P_{R M}$ cannot be occupied if $O_{R 3}$ is occupied. RNA polymerase is bound to $P_{R}$ only for any of the states $s \in\{28,29,30\}$, to $P_{R M}$ only for $s \in\{31, \ldots, 39\}$, and to both for the state $s=40$. The protein and dimer concentrations

$$
x=[\mathrm{CI}], \quad y=[\mathrm{Cro}], \quad u=\left[\mathrm{CI}_{2}\right] \quad \text { and } \quad v=\left[\mathrm{Cro}_{2}\right]
$$

evolve via the chemical rate equations

$$
\begin{aligned}
& \frac{d x}{d t}=k_{x} I_{x}(s(t))-k_{d x} x-2 k_{1}^{r} x^{2}+2 k_{-1}^{r} u, \\
& \frac{d y}{d t}=k_{y} I_{y}(s(t))-k_{d y} y-2 k_{1}^{c} y^{2}+2 k_{-1}^{c} v, \\
& \frac{d u}{d t}=k_{1}^{r} x^{2}-k_{-1}^{r} u, \\
& \frac{d v}{d t}=k_{1}^{c} y^{2}-k_{-1}^{c} v,
\end{aligned}
$$

where $I_{x}(s)$ and $I_{y}(s)$ are indicator functions equal to 1 if $s \in \mathcal{S}_{x}=\{31,32, \ldots, 40\}$ or $\left.s \in \mathcal{S}_{y}=\{28,29,30,40\}\right)$ respectively, and 0 otherwise. ${ }^{7}$

The state $s(t+\Delta t)$ is drawn randomly from a distribution

$$
\mathbf{p}(t+\Delta t)=e^{A(u(t), v(t)) \Delta t} \mathbf{p}(t),
$$

where $A(u, v)$ is a dimer concentration dependent matrix of propensities taking the form of Eq. (7.2) and $p_{r}(t)=\delta_{r s(t)}$ is the probability distribution concentrated at the state $s(t)$.

\subsubsection{Parameter Values: Chemical Rate Constants}

In Table 7.1 are listed our values for the chemical rate constants occurring in Eqs. (7.8). Protein monomer production is a complex process involving transcription of DNA to mRNA which is then translated to proteins, which we model by effective rate constants $k_{x}$ and $k_{y}$. Our model is similar to the phage $\lambda$ switch model of Reinitz and

${ }^{7}$ This model can be further refined by replacing each indicator function by an improved estimate of the expected occupancy fraction $\sum_{s \in \mathcal{S}_{x}} \int_{t}^{t+\Delta t} p_{s}(\tau) d \tau / \Delta \tau$, and similarly for $y$. 
Table 7.1. Chemical rate constants for the hybrid model of the phage $\lambda$ switch.

\begin{tabular}{lll}
\hline Parameter & \multicolumn{1}{c}{ Value } & \multicolumn{1}{c}{ Source } \\
\hline$k_{x}$ & $1.1 \mathrm{nM} \mathrm{s}^{-1}$ & Table 2 of Ref. [13] \\
$k_{y}$ & $0.5 \mathrm{nM} \mathrm{s}^{-1}$ & Adjusted \\
$k_{d x}$ & $7.0 \times 10^{-4} \mathrm{~s}^{-1}$ & Table 3 of Ref. [5] \\
$k_{d y}$ & $2.5 \times 10^{-3} \mathrm{~s}^{-1}$ & Table 3 of Ref. [5] \\
$k_{1}^{r}$ & $9.0 \times 10^{-2} \mathrm{nM}^{-1} \mathrm{~s}^{-1}$ & Table 2 of Ref. [16] and $k_{-1}^{r}$ \\
$k_{-1}^{r}$ & $0.5 \mathrm{~s}^{-1}$ & Table 3 of Ref. [5] \\
$k_{1}^{c}$ & $1.5 \times 10^{-3} \mathrm{nM}^{-1} \mathrm{~s}^{-1}$ & Table 1 of Ref. [17] and $k_{-2}^{r}$ \\
$k_{-1}^{c}$ & $0.5 \mathrm{~s}^{-1}$ & Table 3 of Ref. [5] \\
\hline
\end{tabular}

Vaisnys [13], and we use their value for the CI production rate $k_{x}$. However, we find that their value of $0.078 \mathrm{nM} \mathrm{s}^{-1}$ for the Cro monomer production does not produce sufficient quantities of Cro proteins to establish a lytic phase within our simulations. Instead, we have chosen a higher value of $0.5 \mathrm{nM} \mathrm{s}^{-1}$, which is of the same order as the CI production rate. For the protein monomer decay rates we take values of the more sophisticated stochastic simulation model of Arkin et al. [5].

Dimerisation equilibrium constants are well established for CI from the work of Burz et al. [16], who give $k_{1}^{r} / k_{-1}^{r}=1.8 \times 10^{8} \mathrm{M}^{-1}$, and for Cro from the work of Darling et al. [17], who give $k_{1}^{c} / k_{-1}^{c}=3.07( \pm 1.08) \times 10^{6} \mathrm{M}^{-1}$. For the backward reaction rates we use the Arkin [5] value of $k_{-1}^{r}=k_{-1}^{s}=0.5 \mathrm{~s}^{-1}$ and infer forward rates from the equilibrium constants.

\subsubsection{Parameter Values: Propensities}

A series of papers by Ackers and co-workers beginning with Ref. [18] and culminating in Darling et al. [19] measure equilibrium binding free energies for the 40 states representing the possible binding configurations of $\mathrm{CI}$ and Cro dimers to operators and RNA polymerase to the promoters $P_{R}$ and $P_{R M}$. As we explain below, these can give us ratios of forward to backward reaction rates for binding, though not a complete specification of the rates themselves.

Following Ref. [19], the equilibrium probablility of configuration $s$ at temperature $T$ is given in terms of the dimensionless binding free energy $\Delta G_{S} /(R T)$ by the Gibbs distribution

$$
f_{s}=\frac{C_{s} \exp \left(-\Delta G_{s} / R T\right) u^{i(s)} v^{j(s)}[\mathrm{RNAP}]^{k(s)}}{\sum_{r=1}^{40} C_{r} \exp \left(-\Delta G_{r} / R T\right) u^{i(r)} v^{j(r)}[\mathrm{RNAP}]^{k(r)}},
$$

where $u$ and $v$ are the CI and Cro dimer concentrations, [RNAP] is the concentration of RNA polymerase and $i(s), j(s)$ and $k(s)$ are the number of CI dimer, Cro dimer and 
RNAP molecules bound to the DNA in state $s$. For each state we have included a preexponential factor $C_{s}$ of dimension (concentration) ${ }^{-[i(s)+j(s)+k(s)]}$ to ensure that each term in the sum in the denominator is of the same dimension. This factor is not present in the papers of Ackers and co-workers, nor in the work of Refs. [13] and [10] who use the same formula. Reading between the lines, it appears that all previous authors have implicitly used $C_{s}=(1 \mathrm{M})^{-[i(s)+j(s)+k(s)]}$, and we shall use the same assumption here.

We are interested in estimating the propensities occurring in the discrete state master equation (7.9). It is sufficient to include only transitions in which a single dimer or polymerase attaches to or detaches from the DNA, as the probability of more than one such event occurring in time $d t$ is of $O\left(d t^{2}\right)$. Thus, every possible transition is described by a process of the form

$$
R+O \underset{k_{\mathrm{b}}}{\stackrel{k_{\mathrm{f}}}{\rightleftharpoons}} R O,
$$

where $R$ represents a dimer or polymerase and $O$ the operator or promoter to which it binds. In the case of the phage $\lambda$ switch with its 40 binding states there are 82 such possible processes. Consider two states $r$ and $s$ which differ only in that $s$ is the state $r$ with one extra dimer or polymerase attached. If we denote the propensity for the attachment transition $r \rightarrow s$ as $\alpha_{r s}$ and that for the detachment transition $s \rightarrow r$ as $\beta_{s r}$, then in terms of the chemical reaction rates defined above we have

$$
\begin{aligned}
\alpha_{r s} & =k_{\mathrm{f}}[R]=C_{\mathrm{f}} e^{-E_{\mathrm{f}} /(R T)}[R], \\
\beta_{s r} & =k_{\mathrm{b}}=C_{\mathrm{b}} e^{-E_{\mathrm{b}} /(R T)},
\end{aligned}
$$

where $[R]=u, v$ or $[\mathrm{RNAP}]$. Here we have assumed the usual Arrhenius form for chemical rate constants in terms of activation energies $E_{\mathrm{f}}$ and $E_{\mathrm{b}}$. Note that both $\alpha_{r s}$ and $\beta_{s r}$ have the correct dimensions of (time) $)^{-1}$. Consistency with Eq. (7.10) in the equilibrium distribution gives

$$
\frac{\alpha_{r s}}{\beta_{s r}}=C e^{-\Delta G_{s r} /(R T)}[R],
$$

where $\Delta G_{s r}=E_{\mathrm{f}}-E_{\mathrm{b}}=\Delta G_{s}-\Delta G_{r}$, and $C=C_{\mathrm{f}} / C_{\mathrm{b}}=C_{s} / C_{r}=1 \mathrm{M}^{-1}$.

Note that we do not know either $\alpha_{r s}$ or $\beta_{s r}$ individually, so some estimate of one or the other must be made. Aurell et al. [7] argue that, under the assumption of diffusion-limited aggregation, the association time (i.e., the inverse of the forward propensity) of a single molecule can be estimated from the Smoluchowski equation: $\alpha_{r s}{ }^{-1}=V /(4 \pi D L)$. Assuming diffusion-limited aggregation into a region of length $L=5 \mathrm{~nm}$, a bacterial volume of $V=2 \times 10^{-15} 1$ and a diffusion coefficient of $D=5 \mu \mathrm{m}^{2} \mathrm{~s}^{-1}$ give an aggregation time of $\sim 6 \mathrm{~s}$ at a concentration of $0.8 \mathrm{nM}$, or a forward reaction rate of $k_{\mathrm{f}}=\alpha_{r s} /[R] \approx 0.5 \mathrm{nM}^{-1} \mathrm{~s}^{-1}$. This figure is slightly higher than the diffusion-limited prediction of Berg et al. [20]. However, Berg et al. go on to say that measured values of protein binding rates can exceed $k_{\mathrm{f}}=10 \mathrm{nM}^{-1} \mathrm{~s}^{-1}$, and explain these higher values as the effect of a two-step process in which the protein first 
binds to a nearby non-specific site, and then either slides along the DNA or is directly transferred from one segment of DNA to another. Using these values as a guide, for all forward propensities we have used $\alpha_{r s}=1 \mathrm{nM}^{-1} \mathrm{~s}^{-1} \times[R]$, where $[R]$ is the concentration of the aggregating molecule. Backward propensities $\beta_{s r}$ are then inferred from Eq. (7.13) using binding energies $\Delta G$ from Table 4 of Ref. [19] and a temperature of $T=37^{\circ} \mathrm{C}$, or $R T=0.616 \mathrm{kcal}$.

The RNA polymerase concentration is taken to be [RNAP] $=30 \mathrm{nM} \mathrm{[5].}$

\subsection{Numerical Simulation}

Fig. 7.2 shows the results of our simulation of the phage $\lambda$ switch. The top panel shows the binding of RNA polymerase to the promoters $P_{R M}$ and $P_{R}$ and binding of $\mathrm{CI}_{2}$ and $\mathrm{Cro}_{2}$ dimers to the three intervening operators. The bottom panel shows the concentrations of the proteins and dimers.

For the period $t=0$ to $t=1000$ seconds, parameter values are those listed in the previous section, and the algorithm described at the end of Section 7.2 evolves over time steps of $0.2 \mathrm{~s}$. Over this period $\mathrm{CI}_{2}$ is bound to $O_{R 1}$ and $O_{R 2}$ for most of the time and Cro production is repressed. There is binding of $\mathrm{Cro}_{2}$ to $\mathrm{O}_{R 3}$, but not enough to prevent production of CI. In the lower panel we see steady concentrations of $\mathrm{CI}_{2}$ and $\mathrm{Cro}_{2}$ dimers, with values typical of the lysogenic state [5].

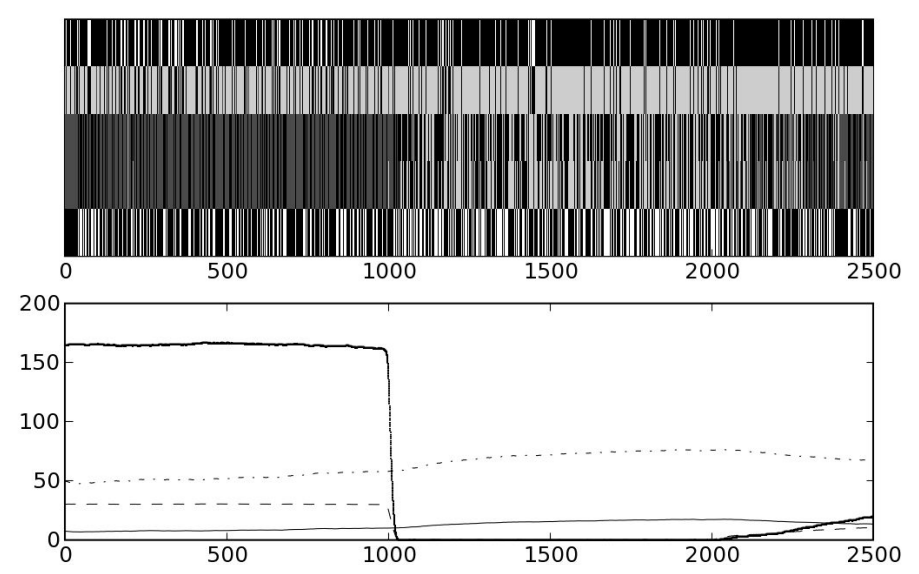

Fig. 7.2. Simulation of the evolution of the phage $\lambda$ switch. Top panel: The five bands represent (from top to bottom) $P_{R M}, O_{R 3}, O_{R 2}, O_{R 1}$ and $P_{R}$ sites with time in seconds running along the horizontal axis. RNA polymerase (white) binds to promoters $P_{R M}$ and $P_{R} \cdot \mathrm{CI}_{2}$ (dark grey) and $\mathrm{CrO}_{2}$ (light grey) dimers bind to operators $O_{R 3}, O_{R 2}$ and $O_{R 1}$. Unoccupied sites are shown in black. Bottom panel: Concentration in $\mathrm{nM}$ of $\mathrm{CI}$ (dashed line) and Cro (dash-dot line) monomers and $\mathrm{CI}_{2}$ (thick line) and $\mathrm{Cro}_{2}$ (thin line) dimers. The horizontal axis is the time in seconds. The effect of UV light on CI monomers is simulated by increasing the degradation rate $k_{d x}$ to $1 \mathrm{~s}^{-1}$ between $t=1000 \mathrm{~s}$ and $t=2000 \mathrm{~s}$. 
AU: Should this be $t=2000$ ?
When phage $\lambda$ infected bacteria are exposed to UV light, a protein called RecA is activated. This protein has the effect of cleaving the $\mathrm{CI}_{2}$ dimers, rendering them unable to bind to operators [1]. We have simulated this by increasing the CI degradation rate $k_{d x}$ from its previous value of $7.0 \times 10^{-4} \mathrm{~s}^{-1}$ to $1 \mathrm{~s}^{-1}$ over the period from $t=1000 \mathrm{~s}$ to $t=2000 \mathrm{~s}$. As the $\mathrm{CI}_{2}$ concentration drops, $O_{R 1}$ and $O_{R 2}$ are freed up. There is some binding of $\mathrm{CrO}_{2}$ to these operators, but not enough to prevent an increase in production of Cro, causing the system to move towards the lysis. As the UV light is switched off at $t=3000 \mathrm{~s}$ the system begins to return to lysogeny.

\subsection{Conclusion}

The ultimate aim of mathematical models of regulatory networks is to be predictive rather than descriptive. Analyses of stability, bistability and robustness may be possible once one has a sound model of the system, usually based upon stochastic processes and differential equations. The stochastic master equation can be used to model the evolving probability distribution of a system over the entire state space, or it can be used to simulate typical trajectories of single cells through state space. The eventual aim is to provide models that can lead back into experiment by predicting the proportions of sub-populations which have followed particular paths, by predicting the upper and lower limits of unknown pathway parameters and rates, by modelling the behaviour of systems under perturbation or by providing the quantitative reasoning behind existing biological systems.

In this chapter we have presented a step on the road to this eventual aim: a hybrid model of gene regulation in which gene switching is dealt with probabilistically using the stochastic master equation while protein and substrate concentrations are dealt with deterministically using classical chemical rate equations. The model deals with moderately complex biological systems economically by reducing the dimension of the phase space to manageable proportions without losing the probabilistic nature of the system.

We have applied the model to perhaps the best-known simple but realistic genetic regulatory system: the switching mechanism between lysogeny and lysis of the Bacteriophage $\lambda$. We have tried to stick as closely as possible to empirically measured physical input parameters with minimal adjustment. The model moves between lysogeny and lysis over realistic time scales as the host bacterium is stressed by the application of ultraviolet light.

This model is not yet at the stage of being predictive. Further refinements of our generic hybrid model are possible, and the next stage will be to implement them. Gene transcription and translation is currently modelled as a single-step process with a single rate parameter. We next intend to include mRNA as an intermediate step and introduce a time delay to account for the transcription step. 


\section{References}

1. Ptashne, M.: A Genetic Switch: Phage $\lambda$ and Higher Organisms (second edition). Cell Press, Cambridge, Massachusetts (1992).

2. de Jong, H.: Modeling and simulation of genetic regulatory systems: a literature review. J. Comp. Biol., 9, 67-103 (2002).

3. Booth, H.S., Burden, C.J., Hegland, M., Santoso, L.: Markov process modelling of gene regulation. Austr. Math. Soc. Gazette, 32, 31-41 (2005).

4. Hasty, J., McMillen, D., Isaacs, F., Collins, J.J.: Computational studies of gene regulatory networks: in numero molecular biology. Nature, 2, 268-278 (2001).

5. Arkin, A., Ross, J., McAdams H.H.: Stochastic kinetic analysis of developmental pathway bifurcation in phage $\lambda$-Infected Escherichia coli Cells. Genetics, 149, 1633-1648 (1998).

6. Kepler, T.B., Elston T.C.: Stochasticity in transcriptional regulation: origins, consequences, and mathematical representations. Biophys. J., 81, 3116-3136 (2001).

7. Aurell, E., Brown, S., Johanson, J., Sneppen, K.: Stability puzzles in phage $\lambda$. Phys. Rev. E., 65, 051914 (2002).

8. Judd E.M., Laub, M.T., McAdams H.H.: Toggles and oscillators: new genetic circuit designs. BioEssays, 22, 507-509 (2000).

9. Elowitz M.B., Lelbler S.: A synthetic ocsillatory network of transcriptional regulators. Nature, 403, 335-338 (2000).

10. Tian, T., Burrage, K.: Bistability and switching in the lysis/lysogeny genetic regulatory network of bacteriophage $\lambda$. J. Theor. Biol., 227, 229-237 (2004).

11. van Kampen, N.G.: Stochastic Processes in Physics and Chemistry. North-Holland, New York, USA (1981).

12. McAdams, H.H., Arkin, A.: Stochastic mechanisms in gene expression. Proc. Natl. Acad. Sci. USA, 94, 814-819 (1997).

13. Reinitz, J., Vaisnys, J.R.: Theoretical and experimental analysis of the phage lambda genetic switch missing levels of co-operativity. J. Theor. Biol., 145, 295-318 (1990).

14. Hasty, J., Isaacs, F., Dolnik, M., McMillen, D., Collins, J.J.: Designer gene networks: Towards fundamental cellular control. Chaos, 11, 207-220 (2001).

15. Gillespie, D.T.: A general method for numerically simulating the stochastic time evolution of coupled chemical reactions. J. Comput. Phys., 22 403-434 (1976).

16. Burz, D.S., Beckett, D., Benson, N., Ackers, G.K.: Self assembly of bacteriophage $\lambda$ CI repressor: Effects of single site mutations on monomer-dimer equilibrium. Biochemistry, 33, 8399-8405 (1994).

17. Darling, P.J., Holt, J.M., Ackers, G.K.: Coupled energetics of $\lambda$ cro repressor self-assembly and site-specific DNA operator binding I: Analysis of cro dimerisation from nanomolar to micromolar concentations. Biochemistry, 33, 11500-11507 (2000).

18. Ackers, G.K., Johnson, A.D., Shea, M.A.: Quantitative model for gene regulation by $\lambda$ phage repressor. Proc. Nat. Acad. Sci., 79, 1129-1133 (1982).

19. Darling, P.J., Holt, J.M., Ackers, G.K.: Coupled energetics of $\lambda$ cro repressor self-assembly and site-specific DNA operator binding II: Cooperative interactions of cro dimers. J. Mol. Biol., 139, 163-194 (2000).

20. Berg, O.G., Winter, R.B., von hippel, P.H.: How do genome-regulatory proteins locate their DNA target sites? Trends Biochem., 7, 52-55 (1982). 